\title{
Die Pflicht zur Leistung von Notfalldienst durch Ärzte in der Praxis
}

\author{
Der ärztliche Notfalldienst wird im ambulanten Bereich meist durch Ärzte mit Privat- \\ praxis erbracht. Die Berufsverbände sind mit der Organisation betraut. Dies ähnelt \\ einer Übertragung staatlicher Aufgaben an Private, daraus folgt nach Meinung des \\ Autors ein Entschädigungsanspruch der Berufsverbände gegenüber den Kantonen.
}

Simon Graf

Korrespondenz:

Dr. med. Simon Graf, BLaw Facharzt FMH

für Allgemeinmedizin Dorf 340

CH-9035 Grub

simon.graf[at]hin.ch
Laut FMH-Statistik arbeiten von den 30273 Ärzten in der Schweiz 53\% im ambulanten Bereich. Davon beteiligen sich gut die Hälfte an einem Grundversorger-Notfalldienst, knapp ein Drittel an einem spezialärztlichen Notfalldienst [1]. Die zeitliche Belastung durch den Dienst schwankt erheblich, je nach Dienstregion fallen zwischen 4 bis 186 Tage Dienst auf einen einzelnen Arzt. Abgesehen von der aufgewendeten Zeit ist häufig auch die Tätigkeit an sich belastend, so dass der Notfalldienst (NFD) für Ärzte in der Praxis ein Dauerthema ist [2].

In der juristischen Lehre und der Rechtsprechung in der Schweiz ist der ärztliche NFD ein Randgebiet. Dieser Beitrag soll deshalb eine Übersicht über die rechtliche Seite des NFD der Ärzte in der Praxis geben. Nach Erläuterung der gesetzlichen Grundlagen auf Bundes- und Kantonsebene folgen Ausführungen zu den durch den NFD tangierten Grundrechten der Ärzte. Kurz beschrieben wird die praktische Ausgestaltung der kantonalen Notfalldienstpflicht durch die Berufsverbände, abschliessend folgen Überlegungen zu Entschädigungsfragen.

\section{Gesetzliche Grundlagen auf Bundesebene}

Aufgrund der Ermächtigung in Art. 95 der Bundesverfassung der Schweizerischen Eidgenossenschaft (BV) zum Erlass von Vorschriften über die Ausübung privatwirtschaftlicher Tätigkeiten hat der Bund 2007 das Bundesgesetz über die universitären Medizinalberufe (Medizinalberufegesetz, MedBG) in Kraft gesetzt. Art. 40 lit. g MedBG lautet: «Sie (Personen, die einen universitären Medizinalberuf selbständig ausüben) leisten in dringenden Fällen Beistand und wirken nach Massgabe der kantonalen Vorschriften in Notfalldiensten mit.» Das MedBG gibt den Kantonen den Auftrag, Behörden zur Aufsicht über die Tätigkeit der Medizinalpersonen zu bilden (Art. 41 MedBG) und gibt ihnen Disziplinarmassnahmen bei Verletzung der Berufspflichten in die Hände (Art. 43 MedBG). Die Disziplinarmassnahmen reichen von Verwarnung über

\section{Le devoir des médecins installés de} participer à un service d'urgence

Dans le domaine ambulatoire, les services d'urgence sont depuis longtemps assurés principalement par les médecins installés. Les législations cantonales mais plus généralement la loi fédérale sur les professions médicales (LPMéd) stipulent, en plus des obligations déontologiques, le devoir des médecins de participer à un tel service. Suivant les cantons, les associations professionnelles de médecins sont directement ou indirectement mandatées pour l'organisation des services d'urgence.

En revanche, le devoir de participer à un service d'urgence foule aux pieds les principes fondamentaux de la liberté économique (art. 27 al. Cst.) et de la liberté individuelle (art. 10 al. 2 Cst.). Mais conformément à I'art. 36 Cst., ces restrictions ont force légale: reconnues d'intérêt général, elles reposent sur une loi et répondent au principe de proportionnalité.

Le devoir de participer à un service d'urgence a une double nature juridique. Il s'agit d'une part d'un devoir imposé directement à chaque médecin qui est rémunéré par une indemnité forfaitaire calculée en fonction de l'heure à laquelle se déroule l'intervention d'urgence. D'autre part, l'organisation des services d'urgence est confiée aux associations professionnelles. Légalement, cette constellation ressemble en plusieurs points à un transfert de tâches étatiques à des organisations privées, ce qu'un arrêt récent du Tribunal fédéral confirme. L'auteur pense par conséquent que les associations professionnelles peuvent prétendre à un dédommagement de la part des cantons pour les dépenses liées à I'organisation des services d'urgence. 
Busse bis zu befristeten oder definitiven Berufsausübungsverboten.

\section{Kantonale gesetzliche Grundlagen}

Die in Art. 40 lit. g MedBG erwähnte Berufspflicht zur Beteiligung an Notfalldiensten gilt nur im Zusammenhang mit einer im Kanton der Praxisausübung gültigen gesetzlichen Grundlage. Aufgrund des MedBG alleine würde keine Notfalldienstpflicht bestehen. Die Kantone der Schweiz haben regelmässig ein Gesundheitsgesetz mit entsprechenden Verpflichtungen der Ärzte zum Notfalldienst. Exemplarisch dafür steht der Kanton Zürich mit $§ 17$ Abs. 1 Gesundheitsgesetz (GesG $\mathrm{ZH})$ :«Ärztinnen und Ärzte (...) sind verpflichtet, Notfalldienst und in dringenden Fällen Beistand zu leisten.» gesellschaften (Notfalldienstreglemente) [4]. In diesen Vorschriften nimmt das Thema «Dispensationen und Ersatzabgaben» einen entsprechend grossen Raum ein. Im Gegensatz dazu bestehen in kleineren Kantonen mit weniger hochspezialisierten Ärzten entsprechend einfachere Reglemente bezüglich Dispensationen und Ersatzabgaben. So beispielsweise in Appenzell Ausser- und Innerrhoden, wo das entsprechende Reglement der den NFD organisierenden kantonalen Ärztegesellschaft lediglich zwei Seiten umfasst.

\section{Verfassungsmässige Einschränkung der Grundrechte des Arztes}

Die Verpflichtung zu einem Notfalldienst rund um die Uhr schränkt zwei durch die Bundesverfassung zugesicherte Grundrechte (Freiheitsrechte)

\section{Die Verpflichtung zum Notfalldienst tangiert die Grundrechte der Wirtschaftsfreiheit und der persönlichen Freiheit.}

Die Organisation des Notfalldienstes wird regelmässig den Ärzten selbst zugewiesen. Dabei gibt es kantonale Unterschiede: Im Kanton Zürich heisst es «Kanton und Gemeinden sorgen für eine zweckmässige Organisation der Notfalldienste, wo solche nicht bestehen.» (§ 17 Abs. 2, 1. Satz GesG ZH). Der Staat würde hier somit subsidiär tätig.

Im Gegensatz dazu wird beispielsweise in den Kantonen Appenzell Ausserrhoden und Innerrhoden die Organisation der Notfalldienste explizit den Berufsverbänden zugewiesen (Art. 42 Abs. 1 Gesundheitsgesetz Appenzell Ausserrhoden [GesG AR] resp. Art. 16b Abs. 1 Gesundheitsgesetz Appenzell Innerrhoden [GesG AI]).

\section{Praktische Ausgestaltung der Dienstpflicht durch die Berufsorganisationen}

Wie oben erwähnt, ist die Organisation des NFD durch direkte oder indirekte Zuweisung durch kantonale Gesundheitsgesetze den Ärzten überlassen. Konkret sind dies die Ärztegesellschaften der entsprechenden Kantone. Im Grosskanton Zürich wird diese Aufgabe zusätzlich an Bezirksgesellschaften delegiert.

Aufgabe der Berufsorganisationen ist neben der eigentlichen Organisation und Planung der Dienste insbesondere auch das Dispensationswesen, wobei gerade in diesem Bereich die rechtsgleiche Behandlung aller am NFD Beteiligten wichtig ist. Im Kanton Zürich existieren dazu ausführliche Regelungen auf Ebene der kantonalen Ärztegesellschaft (Rahmenvorschriften für die Organisation des Notfalldienstes im Kanton Zürich der ÄrzteGesellschaft des Kantons Zürich AGZ) [3] und auch Regelungen der 6 Bezirks- der Ärzte ein: die Wirtschaftsfreiheit (Art. 27 BV) und die persönliche Freiheit (Art. 10 BV). Die Wirtschaftsfreiheit ist beispielsweise dadurch eingeschränkt, dass ein Arzt im Dienst u. U. jemanden gegen seinen Willen behandeln muss. Die persönliche Freiheit wird tangiert durch die Einschränkung der Freizeit (Lebensgestaltung) wegen der Leistung von Notfalldienst.

Einschränkungen von Grundrechten, genauer der Freiheitsrechte, sind möglich. Die Einschränkungen müssen sich aber an die Erfordernisse von Art. 36 BV halten: Sie müssen eine gesetzliche Grundlage aufweisen, es muss ein öffentliches Interesse dafür bestehen und die Einschränkungen haben verhältnismässig zu sein. Zudem muss der Kerngehalt des Grundrechtes gewahrt bleiben.

Das öffentliche Interesse liegt darin, dass in ländlicher Umgebung ohne naheliegendes Spital zur Sicherung der öffentlichen Gesundheit und Sicherheit nur die Dienstleistung durch Ärzte in Frage kommt. In städtischen Gebieten dienen die ärztlichen Notfalldienste der Entlastung der Notfallambulatorien der Spitäler, damit diese ihren Kernauftrag (Behandlung von spitalbedürftigen Notfällen) besser erfüllen können.

Die Verhältnismässigkeit der Einschränkung kann ebenfalls bejaht werden: Die Notfalldienste sind geeignet für die Versorgung der Bevölkerung. Sie sind erforderlich, da keine anderen Medizinalpersonen aufgrund ihrer Fachkenntnisse eine solche Dienstleistung übernehmen können. Die Abwägung der Grundrechtseinschränkung auf der Arzt-Seite mit dem Recht auf medizinische Behandlung (Art. 10 BV Recht auf Leben und persönliche Freiheit) der Bevölkerung 


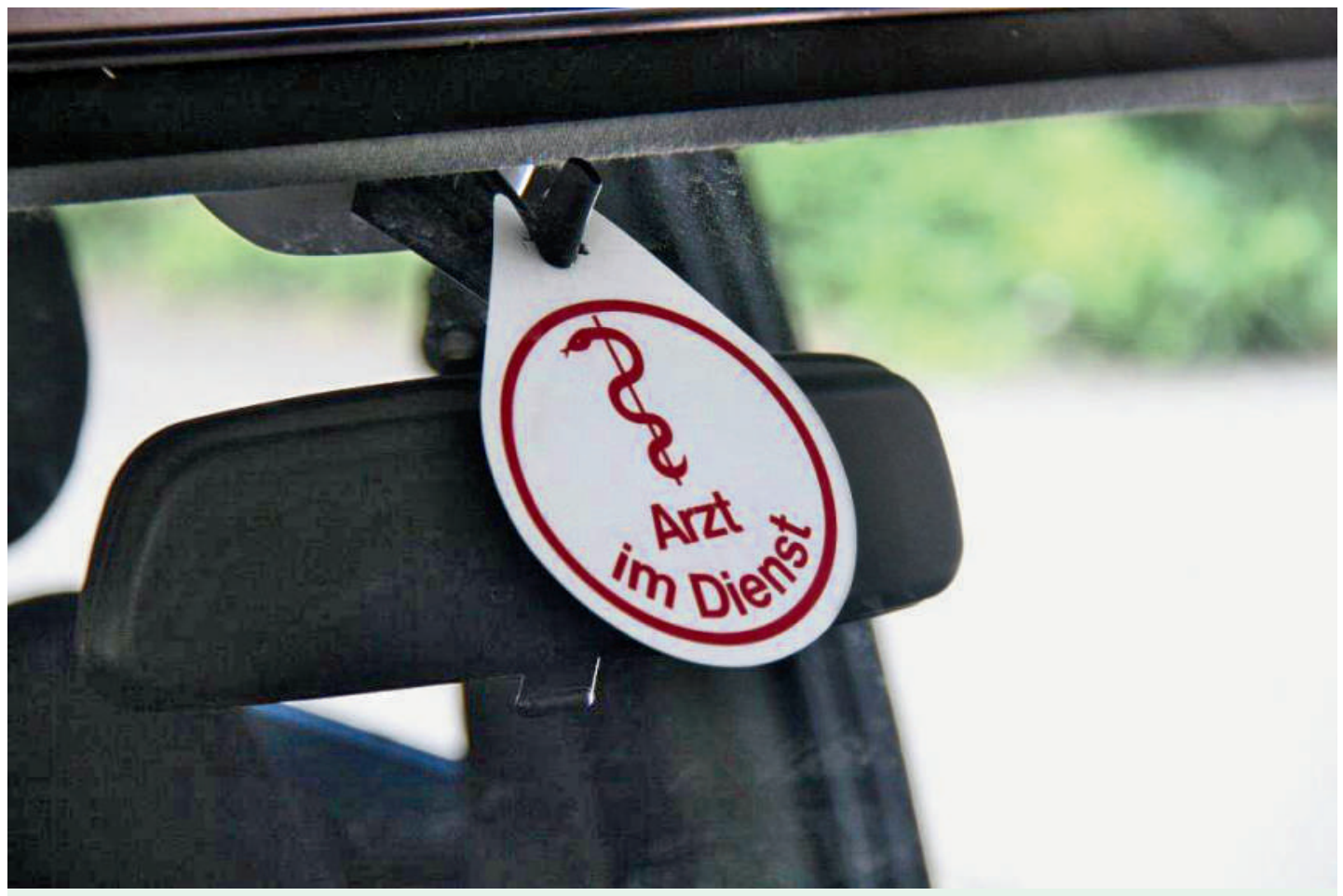

Stets im Einsatz - für den Notfalldienst muss immer ein Arzt zur Verfügung stehen. Die Organisation obliegt den Berufsverbänden.

wird ferner zugunsten der Gesundheit der Bevölkerung ausfallen. Zudem hat der Gesetzgeber nicht nur das mildeste, sondern schlicht das - insbesondere in ländlichen Gebieten - einzige Mittel gewählt, dieses Ziel zu erreichen, nämlich die Verpflichtung des einzelnen Arztes zur Leistung von Notfalldienst [5].

\section{Rechtsnatur der Verpflichtung zum Notfalldienst}

Die Rechtsnatur der Verpflichtung zum Notfalldienst ist zweigeteilt:

Einerseits ist sie eine einem Individuum (dem Arzt, der Ärztin) auferlegte Pflicht (ähnlich Wehrpflicht, Feuerwehrpflicht, Amtszwang). Diese wird als verwaltungsrechtliche Pflicht bezeichnet, die unmit- gabe an Private übertragen. Beleihungen werden in der Regel über einen verwaltungsrechtlichen Vertrag vorgenommen. Zudem ist bei Beleihungen eine Entschädigung üblich und gegebenenfalls würde auch eine Staatshaftung zum Tragen kommen [7].

Trotz Fehlens einiger dieser Kriterien muss die Delegation der Organisation des NFD an die Ärztegesellschaften als Beleihung bezeichnet werden. Gestützt wird diese Ansicht auch durch ein aktuelles Urteil des Bundesgerichtes vom 25. Oktober 2011 [8]: Das Gericht hatte in diesem Fall die Beschwerde eines vom Notfalldienst dispensierten Arztes aus dem Kanton Thurgau gegen die Erhebung einer Ersatzabgabe zu behandeln. Dabei führt das Bundesgericht aus, dass die gesetzliche Delegation der Organisation des NFD an die

\section{Dieses Rechtsverhältnis weist viele Aspekte einer Beleihung (Übertragung von staatlichen Aufgaben an Private) auf.}

telbar durch Rechtssatz begründet wird [6].

Andererseits besteht die Verpflichtung der Berufsverbände zur Organisation des Dienstes. Dieses Rechtsverhältnis ist nicht einfach einzuordnen, da es keinem der üblichen Wege der Delegation von staatlichen Aufgaben an Private folgt. Am ähnlichsten ist das Rechtsverhältnis einer sog. Beleihung. Durch eine Beleihung wird die Erfüllung einer staatlichen Auf-
Ärztegesellschaften dazu führt, dass diese hoheitlich tätig werden. Damit haben sich die Ärztegesellschaften an die allgemeinen Grundsätze des öffentlichen Rechtes zu halten haben (in diesem Falle insbesondere die Gleichbehandlung der Dienstpflichtigen hinsichtlich Dispensation und Ersatzabgabe). Das Gericht macht damit deutlich, dass es sich bei der Delegation der NFDOrganisation im Prinzip um eine Beleihung handelt. 


\section{Wie werden am Notfalldienst Beteiligte entschädigt?}

Es existieren zwei Bereiche von Entschädigungsfragen: einerseits die ärztliche Tätigkeit im Notfall selbst und andererseits der Aufwand, der mit der Organisation des Notfalldienstes zusammenhängt und somit die Berufsorganisationen betrifft.

Der Arzt rechnet bei einem Notfalleinsatz gemäss TARMED (gesamtschweizerisch gültige Tarifstruktur für ambulante ärztliche Leistungen in Praxis und Spitalambulatorium) ab. Im Notfall erhält der Arzt neben der Grundtaxe für die eigentliche Leistung eine dem Wochentag resp. der Tageszeit angepasste zusätzliche Entschädigung («Notfall-Inkonvenienzpauschale»). Diese Pauschale wird durch den Patienten resp. seine Sozialversicherung getragen. Damit wird dem Arzt die sofortige Einsatzbereitschaft abgegolten («Inkonvenienz»), allerdings sind die Wartezeit/Pikettzeit (Vorhalteleistung) als solche und auch damit zusammenhängende Kosten wie Personalkosten damit nicht finanziert.

Bei den in den Kantonen die Organisation des NFD übernehmenden ärztlichen Berufsorganisationen entsteht eine ganze Anzahl von Aufwendungen: Die Ärztevereine haben die ganze Dienstorganisation aufzuziehen, und sie haben sich mit den Fragen um Dispensationen und Ersatzabgaben $\mathrm{zu}$ befassen. Dann müssen die Diensteinteilungen auf Ebene Dienstkreis von entsprechenden Verantwortlichen geplant werden und schliesslich ist der Aufwand der Publikation (z. B. Inserate in Zeitungen) und auch der telefonischen Erreichbarkeit und Triage (z.B. über ärztliche Notfallzentralen wie das Ärztefon [9]) nicht zu vernachlässigen. In gewissen Kantonen übernehmen Ärztegesellschaften ferner auch einen Teil der Verluste durch nichtbezahlte Notfall-Rechnungen. Für alle diese Kosten werden die Berufsverbände bis anhin nicht entschädigt.

\section{Besteht eine Entschädigungspflicht der Kantone an die Ärztegesellschaften?}

Im Kanton Zürich wird nach den Buchstaben des Gesetzes nur dem einzelnen Arzt eine Verpflichtung zum Notfalldienst auferlegt. Es wird offensichtlich davon ausgegangen, dass der seit jeher durch die Ärztevereinigungen organisierte Dienst so weitergeführt wird. Der Kanton oder die Gemeinden würden erst subsidiär, bei «Versagen» der NFD-Organisation durch die Ärzte aktiv. Da keine wörtliche gesetzliche Übertragung einer Pflicht zur Organisation des NFD an die Ärztegesellschaft besteht, ist es fraglich, ob eine Entschädigung geschuldet ist. Es ist aber nicht zu vergessen, dass laut Art. 13 der Kantonsverfassung des Kantons Zürich (KV ZH) der Staat für die Aufrechterhaltung einer ausreichenden Gesundheitsversorgung zuständig ist. Ferner überträgt § 46 GesG ZH den Gemeinden und dem Kanton eine Verpflichtung zur Unterstützung von Massnahmen zur Gesundheitsförderung. Meines Erachtens kann aus dem sinngemässen Überlassen der NFD-Organisation im GesG und den erwähnten, Kanton und Gemeinden verpflichtenden Vorschriften durchaus eine Verpflichtung zur Finanzierung der NFD-Organisation durch die Berufsverbände abgeleitet werden. Dies, da sich die Delegation - wie oben gezeigt - sehr stark an eine Beleihung anlehnt. Unbegründet ist damit die Ansicht von Poledna/Stoll, dass das Gemeinwesen den Ärzten Kosten für die NFD-Organisation überbinden könnte, falls es aktiv werden müsste bei «Versagen» der Berufsorganisationen. [10]

Andere Gesundheitsgesetze - so etwa in beiden Appenzeller Kantonen - verpflichten nicht nur den einzelnen Arzt zur Leistung von NFD, sondern sie übertragen die Durchführung explizit an die Berufsorganisationen (Art. 16 Abs. 1 GesG AI resp. Art. 42 Abs. 12 . Satz GesG AR). Aufgrund der obenstehenden Überlegungen zur Beleihung (gestützt auch durch den erwähnten Bundesgerichtsentscheid 2C_807/2010) entsteht meines Erachtens bei dieser gesetzlichen Konstellation eine Entschädigungspflicht des Kantons gegenüber den die NFD-Organisation aufrechterhaltenden Berufsorganisationen der Ärzte.

\section{Literatur}

1 Kraft E. 30273 Ärztinnen und Ärzte für die Schweiz. Schweiz Ärztezeitung. 2011;92(12):440-4.

2 von Below G. Organisationsformen und Zeitbelastung im ambulanten ärztlichen Notfalldienst in der Schweiz. In: Arnet B,von Below G. Organisation des ambulanten ärztlichen Notfalldienstes in der Schweiz. Zürich; 2004, S. 3-61.

3 www.aerzte-zh.ch/main.php?typ=mit\&sub=not

4 www.aerzte-zh.ch/main.php?typ=mit\&sub=not

5 Gächter T, Tremp D. Der Arzt und seine Grundrechte. In: Kuhn MW, Poledna T (Hrsg.). Arztrecht in der Praxis. 2. Aufl., Zürich/Basel/Genf; 2007. S. 34.

6 Häfelin U, Müller G, Uhlmann F. Allgemeines Verwaltungsrecht, 6. Aufl., Zürich/St. Gallen; 2010. N 749.

7 Vgl. [6] N 1509 ff.

8 Urteil 2C_807/2010 vom 25. Oktober 2011 der II. öffentlich-rechtlichen Abteilung des Bundesgerichtes.

9 Das Ärztefon ist die offizielle Notfallzentrale der Stadt Zürich und 48 weiterer Gemeinden des Kantons Zürich sowie des Kantons Appenzell Ausserrhoden. 7 Tage pro Woche und rund um die Uhr werden Notfallärzte vermittelt und bei medizinischen Fragen Beratung angeboten. Quelle: Homepage Ärztefon www.aerztefon.ch

10 Poledna T, Stoll R. Ärztlicher Notfalldienst: Pflicht oder Recht des Arztes? - Betrachtungen am Beispiel der Gesundheitsgesetzgebung des Kantons Zürich. In: AJP 11/2005, S. 1367-72. 